\title{
Microstructural Characterization Of Nickel-Based Superalloys
}

\section{E. Sarath K Menon}

\section{UES Inc., 4401 Dayton-Xenia Road, Dayton, OH 45432-1894, USA}

Nickel-based superalloys find extensive use in high-temperature applications. A large number of the alloys used rely on carefully tailored compositions and heat-treatment schedules that result principally in a microstructure constituted of the ordered $\gamma^{\prime}$ phase $\left(\mathrm{L}_{2}\right.$ crystal structure) distributed in a $\gamma$ (FCC crystal structure) matrix. Indeed, the optimization of the requisite properties is achieved by a combination of grain size control, distribution of minority phases such as carbides and borides and most importantly a multi-sized distribution of the $\gamma$ ' phase. Needless to say, a precise knowledge of the uniformity of distribution of the phases, their average sizes as well as their size distribution is essential in evaluating the properties associated with various heattreatments. Here, the advantages of employing a variety of electron microscopy techniques is illustrated and results of quantitative analysis of these microstructures to obtain quantitative parameters such as volume fraction, average size and size distribution of the $\gamma$ ' phase. The use of back-scattered electron imaging in the SEM from thin-foil sections is shown to provide highquality micrographs suitable for accurate quantification of the larger-sized $\gamma$ ' phase (greater than $\sim 50 \mathrm{~nm}$ ), and this technique will be demonstrated.

A variety of Nickel-based superalloys were examined including IN100, René88DT, ME3 under various heat-treatment conditions. A Leica 360FE SEM was employed in all the SEM work described here. TEM was carried out in Phillips CM20 microscope. All TEM foils were prepared by electro-polishing them in a solution containing $35 \mathrm{cc} \mathrm{HClO}_{4}$ in $500 \mathrm{cc}$ methanol and $65 \mathrm{cc} \mathrm{n}$ butanol at $20 \mathrm{~V}$ and a temperature $<-40^{\circ} \mathrm{C}$ Quantitative analysis was carried out using the Image Processing Toolkit plugins for the Adobe Photoshop software. The dark-field images shown in Figure 1 illustrates the typical appearance of the $\gamma^{\prime}$ phase distributed in the $\gamma$ matrix and it can be seen that three size classes of the strengthening precipitate phase are present in this microstructure. These are conveniently referred to as the primary, secondary and the tertiary $\gamma^{\prime}$ phase and their sizes and volume fractions may vary depending upon the alloy compositions and the particular heat-treatment. In order to obtain accurate volume fractions and size distributions of the primary and secondary $\gamma^{\prime}$ phases, it was found that back-scattered electron imaging of thin areas of the TEM foil in the SEM provided the most convenient and rapid method. Figure 2 illustrates several examples of back-scattered electron images obtained from Ni-based superalloys. From these micrographs, it is clear that high-quality images with well-resolved matrix-precipitate images can be obtained very easily from thin areas of the sample. The resolution of these images appear to be remarkably improved due to the smaller volume from which the back-scattered electrons are generated in these thin foils. This renders automation of computer-assisted particle size determination easier and reproducible since thresholding of such images become less prone to errors of judgment. Figure 3 illustrates two examples of particle size distribution curves generated from examination of a large number of back-scattered electron images in the SEM.

The author gratefully acknowledges the support from Air Force contract No. F33615-01-C-5214. 

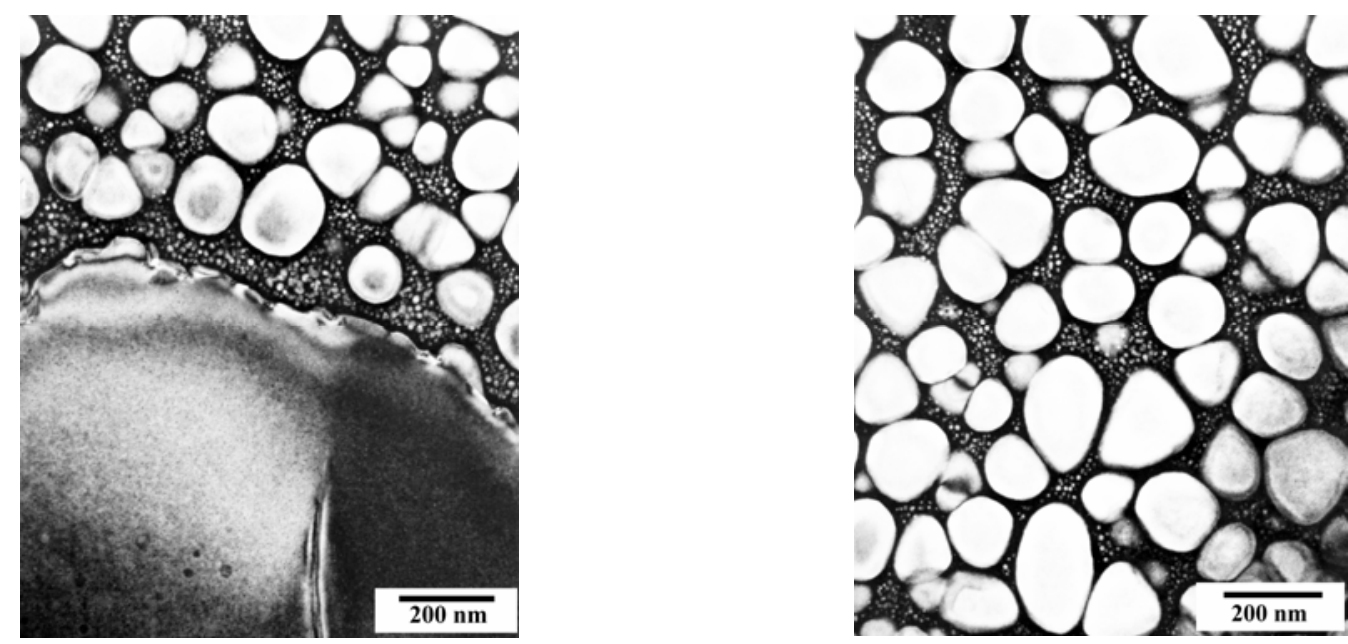

Figure 1: Dark field micrographs from $\mathrm{g}_{110}$ of the $\gamma^{\prime}$ phase showing primary( large precipitate at bottom of left micrograph), secondary (cuboidal particles $\sim 200 \mathrm{~nm}$ in size) and tertiary (fine particles $<20 \mathrm{~nm}$ in size) phases. The foil normals here are [111].

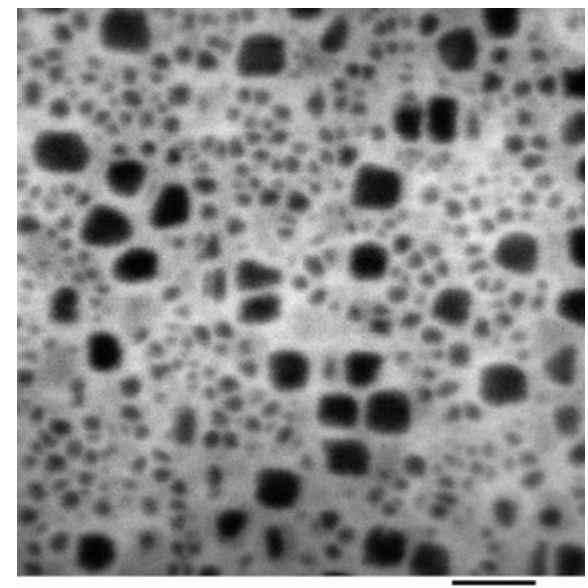

$200 \mathrm{~nm}$

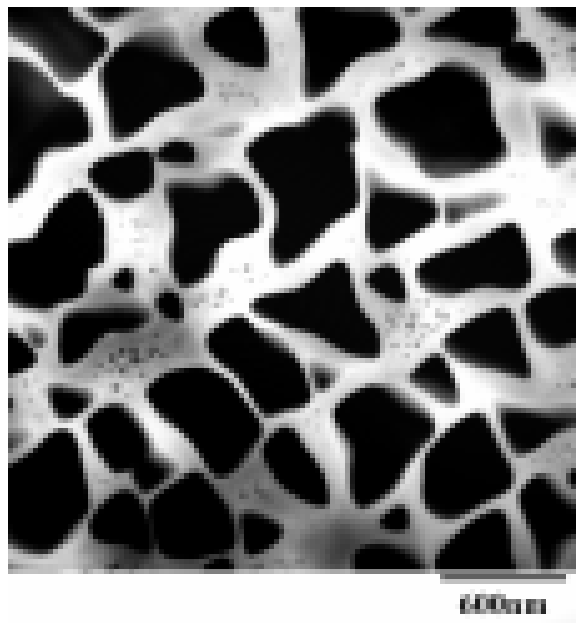

Figure 2 : Back-scattered electron images from SEM showing secondary and tertiary $\gamma^{\prime}$ phases in Ni-base superalloys
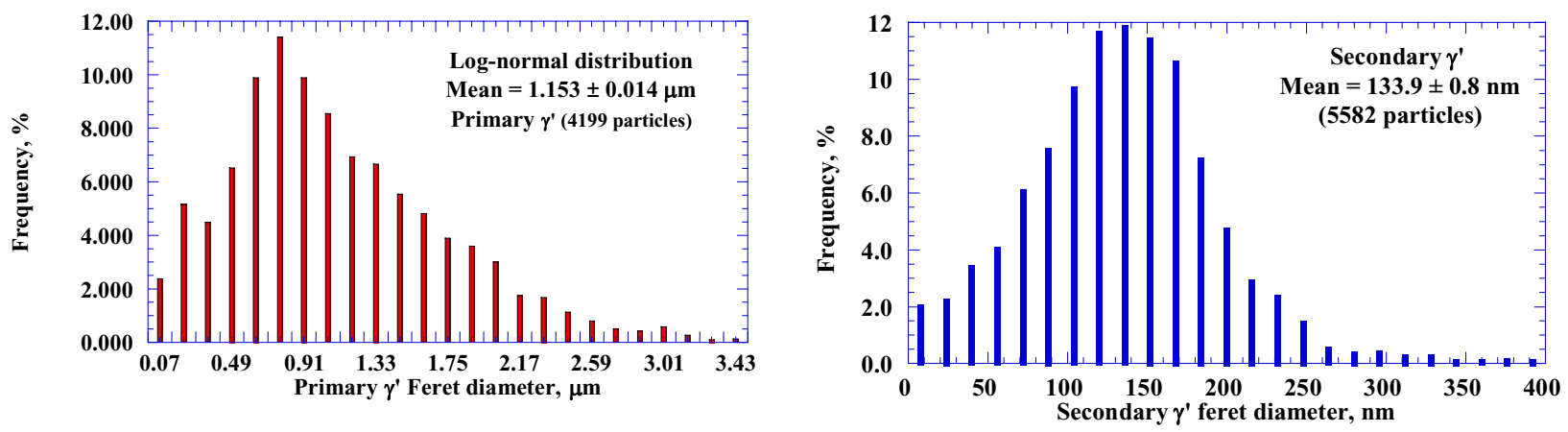

Figure 3: Histogram showing size distribution plots for primary and secondary $\gamma^{\prime}$ phases. 